DESENVOLVMENTO
$\begin{aligned} & \text { SISTEMA } \\ & \text { E MEIO AMBIENTE }\end{aligned}$

\title{
Conflitos e negociações entre a indústria do petróleo e a conservação da Mata Atlântica no Litoral Norte de São Paulo, Brasil
}

\section{Conflicts and Negotiations between Oil Industry and Conservation of the Atlantic Forest in the North Coast of São Paulo, Brazil}

\author{
José Eduardo VIGLIO ${ }^{1 *}$, Jorge CALVIMONTES ${ }^{1}$, Lúcia Costa FERREIRA ${ }^{1}$ \\ ${ }^{1}$ Núcleo de Estudos e Pesquisas Ambientais, Universidade Estadual de Campinas (Unicamp), Campinas, SP, Brasil. \\ *E-mail de contato: eduviglio@hotmail.com
}

Artigo recebido em 8 de junho de 2016, versão final aceita em 4 de outubro de 2017.

RESUMO: Muitas áreas protegidas do planeta estão sob pressão de atividades de mineração e energia. Em relação à exploração e produção de petróleo e gás, existe uma forte correlação entre áreas de alta biodiversidade e formações geológicas que contêm hidrocarbonetos. Além disso, muitas áreas almejadas por esse setor se sobrepõem com áreas sensíveis, ecossistemas ameaçados e territórios indígenas. No Brasil, apesar de a maior parte da exploração e produção de petróleo e gás (cerca de 94\%) ocorrer em alto mar (campos offshore), as atividades de tratamento e transporte ocorrem necessariamente em regiões costeiras e litorâneas. Essa infraestrutura petrolífera tem se instalado nas últimas décadas no altamente ameaçado bioma da Mata Atlântica e em seus ecossistemas costeiros associados. O presente artigo explora o conflito entre a expansão da atividade de petróleo e gás e a conservação da Mata Atlântica do Estado de São Paulo. A partir do conceito de arena, o artigo analisa o processo de licenciamento ambiental do Projeto Mexilhão da Petrobras, envolvendo exploração (offshore), tratamento e transporte de gás natural, no contexto do Parque Estadual da Serra do Mar (PESM) no Litoral Norte de São Paulo. O trabalho destaca o papel e a importância das áreas protegidas como recursos de poder nas negociações. Por si só, a existência dessas áreas pode não ser suficiente para fazer frente às pressões das atividades petrolíferas, mas, sem dúvida, sua presença influencia nas tomadas de decisão e nos resultados dos processos de negociação. Do ponto de vista teórico-metodológico, este trabalho constata a relevância de análises multiatores e multiníveis para tratar do conflito e do processo decisório sobre empreendimentos de energia e a conservação da biodiversidade.

Palavras-chave: conflitos sociais; conservação da biodiversidade; áreas protegidas; Mata Atlântica; petróleo e gás. 
ABSTRACT: Many protected areas of the planet are under pressure from mining and energy activities. In relation to oil and gas exploration and production, there is a strong correlation between areas of high biodiversity and geological formations containing hydrocarbons. Additionally, many areas of interest of the oil industry overlap with sensitive areas, endangered ecosystems, and indigenous territories. In Brazil, although most of the oil and gas exploration and production (around 94\%) are in the offshore fields, the treatment and transportation occur mainly in coastal regions. The oil infrastructure has been installed in the highly threatened Atlantic Forest biome and its associated coastal ecosystems. This paper explores the conflict between the expansion of oil and gas activity and the preservation of the State of São Paulo Atlantic Forest. Based on the arena concept, the paper analyze the environmental licensing process of "Mexilhão Entreprise" of the Brazilian Oil Company (Petrobras) in the State Park of the Serra do Mar (PESM) on the north coast of São Paulo. The paper highlights the role and importance of protected areas as power resources in the negotiations of arena. The existence of these areas may not be enough to deal with the pressures of oil and gas activities, but undoubtedly their presence influences decision-making and the results of negotiation processes. From the theoretical and methodological points of view, this paper shows the importance of multi-actor and multi-level analysis, to deal with the conflict and the decision-making on energy enterprise and biodiversity conservation.

Keywords: social conflicts; conservation of biodiversity; protected areas; Atlantic Forest; oil and gas.

\section{Introdução}

Em nível mundial, segundo dados da IUCN (2008) e da UNESCO (2009), em torno de $25 \%$ das áreas declaradas como patrimônio natural da humanidade estão sob pressão de atividades de mineração e energia. Em relação à mineração, o relatório do World Resources Institute indica que um quarto das áreas atualmente exploradas está localizado em um raio de $10 \mathrm{~km}$ de áreas protegidas categorizadas como I-IV pela IUCN ${ }^{1}$ (Miranda et al., 2003; Carter, 2005; Osti et al., 2011). Um estudo recente de Ferreira et al. (2014) mostrou que, no Brasil, ao menos $20 \%$ da área das unidades de conservação de proteção integral e das terras indígenas estão ameaçadas por interesses de exploração mineral. Do total de 505 unidades de proteção integral analisadas, 236 (ou $47 \%$ do total) têm pedido de pesquisa mineral no Departamento Nacional de Produção Mineral
(DNPM). De 578 terras indígenas, 251 (ou 43\%) estão na mesma situação.

Especificamente em relação às atividades de petróleo e gás, as implicações ecológicas dessa indústria têm sido amplamente documentadas, incluindo impactos diretos e indiretos (Conservation International, 2003; O’Rourke \& Connolly, 2003; San Sebastián \& Hurtig, 2004; Garcia, 2007; Finer et al., 2008; Copeland et al., 2009; Committee on the Effects of the Deepwater Horizon Mississippi Canyon-252, 2013). Embora a indústria de petróleo e gás seja uma atividade de risco em qualquer região, as áreas tropicais são particularmente mais vulneráveis (Carter, 2005). Em razão da alta correlação entre áreas de alta biodiversidade e formações geológicas que contêm hidrocarbonetos, muitas áreas almejadas por essa indústria se sobrepõem com áreas sensíveis, ecossistemas ameaçados e territórios indígenas (Lessmann et al., 2016).

\footnotetext{
${ }^{1}$ A IUCN oferece, desde 1994, um guia para a definição de categorias de áreas protegidas que contém seis categorias de manejo, divididas segundo o grau de proteção. As categorias de I a V são aquelas destinadas à conservação da biodiversidade apenas mediante o uso indireto dos recursos naturais (Dudley, 2008).
} 
São justamente nessas áreas de florestas úmidas que os investimentos da indústria do petróleo vêm crescendo significativamente em razão da descoberta de campos com reservas economicamente viáveis. Estima-se que uma porcentagem significativa de novos empreendimentos em exploração e produção de petróleo e gás nos próximos anos ocorrerá em ambientes sensíveis, como o Alasca e os trópicos úmidos (Leyen, 2008).

Desse modo, é possível inferir a permanência - e mesmo o aumento - dos conflitos entre a exploração de hidrocarbonetos e a conservação da biodiversidade nos próximos anos (Energy \& Biodiversity Initiative, 2003; Carter, 2005; Finer \& Orta-Martinez, 2010; Osti et al., 2011), devido aos seguintes fatores: a) a continuidade da dependência mundial em relação aos combustíveis fósseis (Chow et al., 2003); b) a existência de um novo ciclo de expansão dos combustíveis fósseis em razão da descoberta e exploração do gás de xisto (Fracking) e da intensificação da exploração petrolífera em águas profundas, como a do Pré-sal brasileiro $^{2}$ (O’Rourk \& Connolly, 2003; Maugeri, 2012); e c) a exploração de novas áreas, decorrente do esgotamento de poços maduros (Carter, 2005), cujos dutos e gasodutos podem atravessar vastas áreas protegidas, tanto terrestres quanto marinhas (Jaffe \& Victor, 2004).

Utilizando o conceito de arena como espaço social de interação e decisão que envolve diferentes atores sociais, o presente artigo explora o conflito entre as atividades de petróleo e gás e a conservação da Mata Atlântica do Estado de São Paulo com base na análise do processo de licenciamento ambiental $^{3}$ do Projeto Mexilhão, da Petrobras, no Parque Estadual da Serra do Mar (PESM). Esse empreendimento envolve exploração, tratamento e transporte de gás natural desde os campos offshore até a região onde se encontra o PESM, a maior unidade de conservação de proteção integral do litoral brasileiro e um verdadeiro corredor ecológico, que conecta os mais significativos remanescentes de Mata Atlântica do país, apontada como um dos hotspots mundiais da biodiversidade (Mittermeier et al., 2004). Além da Petrobras, os principais atores dessa arena foram o Instituto Brasileiro de Meio Ambiente e Recursos Naturais Renováveis (IBAMA), o Ministério Público do Estado de São Paulo (MPSP), o Ministério Público Federal (MPF), o Instituto Florestal do Estado de São Paulo (IF) e organizações não governamentais ambientalistas (ONGs). Deslocou-se, portanto, do enfoque genérico e amplo sobre a problemática envolvendo petróleo e conservação para a compreensão do conflito com base em um contexto específico.

Este trabalho aponta e sustenta a relevância do contexto sociopolítico e ambiental para a compreensão do conflito e seus desdobramentos. A existência

\footnotetext{
${ }^{2}$ O petróleo e gás do Pré-sal estão localizados em uma distância que pode chegar a $300 \mathrm{~km}$ da costa, abaixo tanto de uma de lamina d'água, que pode alcançar 5.000 metros de profundidade, quanto de uma camada de sal que pode atingir 3.000 metros. Segundo estudo recente de Jones \& Chaves (2015), as reservas do Pré-sal podem conter cerca de 176 bilhões de barris de petróleo e gás natural. Essas reservas poderão colocar o país entre os dez maiores produtores de petróleo do mundo (Fundação Getúlio Vargas, 2011).

${ }^{3} \mathrm{O}$ licenciamento ambiental é uma obrigação legal prévia à instalação de qualquer empreendimento ou atividade potencialmente poluidora ou degradadora do meio ambiente. As principais diretrizes para a execução do licenciamento ambiental estão expressas na Lei n. 6.938/1981, que estabeleceu a Política Nacional de Meio Ambiente (PNMA), e nas resoluções do Conselho Nacional de Meio Ambiente (CONAMA) n. 001/1986 e n. 237/1997. O processo de licenciamento ambiental apresenta três etapas distintas: licenciamento prévio, licenciamento de instalação e licenciamento de operação.
} 
do PESM, o protagonismo e a atuação dos atores ambientalistas governamentais e não governamentais não foram determinantes para a Petrobras rever a decisão de instalação do empreendimento. No entanto, foram centrais nas negociações para minimização dos impactos ambientais do empreendimento no PESM. O trabalho sustenta ainda que a política de conservação ambiental se torna mais efetiva com a presença e atuação desses atores nas arenas de disputa.

Além dessa introdução, o artigo é composto por outras cinco partes. Na primeira, apresentam-se o recorte teórico-metodológico utilizado e os procedimentos da pesquisa. Em seguida, aborda-se a problemática sobre os conflitos entre a exploração de petróleo e gás e a conservação da Mata Atlântica. Posteriormente, contextualizam-se o Projeto Mexilhão e o Litoral Norte (LN) do Estado de São Paulo. Na quarta parte, apresentam-se os principais resultados e a discussão deste trabalho. Nesse tópico, inicialmente, realiza-se uma descrição da arena do Projeto Mexilhão, com destaque para seus principais atores e respectivos posicionamentos. Posteriormente, com maior ênfase e profundidade, são analisadas e discutidas as disputas e as negociações em torno das implicações ambientais do Projeto Mexilhão no PESM. Por fim, na última parte, seguem-se algumas notas conclusivas sobre o tema abordado e o recorte teórico metodológico utilizado.

\section{Recorte teórico e metodológico}

Para analisar as disputas e as negociações entre os diferentes atores sociais e institucionais do licenciamento ambiental do Projeto Mexilhão, foi utilizada a abordagem teórico-metodológica de arena. Uma arena, nesse sentido, pode ser entendida tanto como uma representação simbólica quanto como um sistema de interação e disputas, um political establishment, que envolve diferentes atores sociais e institucionais com variados interesses e perspectivas, os quais buscam influenciar um processo decisório por meio da mobilização de diferentes recursos sociais (Renn, 1992; Hannigan, 2006; Ferreira, 2012;). Ostrom (2005) usa o conceito físico de holon para tratar a arena como um sistema completo de interação em um nível e que, ao mesmo tempo, é parte de um sistema em outro nível. Desse modo, com base nessa perspectiva de processo decisório em multinível, a arena do licenciamento ambiental do Mexilhão é vista aqui como um sistema de interação e decisão sobre as implicações ambientais do empreendimento, mas também como parte de outras arenas mais amplas que tratam de políticas energéticas e desenvolvimento, por exemplo, as quais não foram objeto de investigação neste trabalho.

A noção de arena possibilitou a consideração tanto de fatores estruturais quanto da dinâmica das interações e estratégias de ação que os atores adotam para influenciar os resultados do processo decisório (Ferreira et al., 2017). Essa abordagem permitiu descortinar diferentes conflitos relacionados ao Projeto Mexilhão. Já o conflito é entendido neste trabalho como "manifestações de clivagens abertas entre dois ou mais atores individuais ou coletivos, que apresentam interesses histórica ou momentaneamente incompatíveis, quanto à apropriação ou controle de bens considerados raros, escassos, sejam materiais ou simbólico" (Ferreira, 2012, p. 3). Os conflitos sociais são vistos aqui muito mais como um fator transformador de práticas sociais e produ- 
tor de mudanças que uma disfunção dos sistemas sociais ou como efeitos patológicos que ameaçam o equilíbrio social (Simmel, 1983; Ferreira, 2005).

O licenciamento ambiental, como principal mecanismo regulatório da questão ambiental de empreendimentos econômicos e de infraestrutura no Brasil, tem sido o instrumento da Política Nacional de Meio Ambiente (PNMA) mais relacionado aos conflitos ambientais no país (Zhouri \& Oliveira, 2007; Agra Filho, 2010; Zhouri \& Laschefski, 2010; Hochstetler, 2011;). Os conflitos e as negociações na arena do licenciamento ambiental do Projeto Mexilhão, especialmente de sua parte terrestre, que envolve a UTGCA e o GASTAU (Processos de licenciamento IBAMA n. 02001.005437/2005-78 e 02001.005436/2005-23), foram tratados por meio de análise documental, entrevistas, observação e registro audiovisual de audiências públicas.

O licenciamento do Projeto Mexilhão teve início em 2005, e as licenças ambientais de operação foram concedidas em 2011. A maioria dos documentos levantados e analisados foi gerada nesse período de 2005-2011 e foi constituída principalmente por pareceres técnicos, gravações em vídeos e atas transcritas de audiências públicas, inquérito civil do MPSP (23/05), Estudo de Impacto Ambiental (EIA) e Relatório de Impacto Ambiental (RIMA) da UTGCA e GASTAU, manifestação e questionamentos técnicos, licenças ambientais (prévia, instalação e operação) e suas respectivas condicionantes. No total, foram levantados e analisados cerca de 60 documentos. A maioria deles foi identificada e levantada no Sistema Informatizado de Licenciamento Ambiental Federal do IBAMA e no Inquérito Civil n. 23/2005 do MPSP sobre o Projeto Mexilhão. Ambas as bases armazenaram e documentaram os dados e as informações relacionados ao processo de licenciamento ambiental analisado. Além disso, foi de fundamental importância a identificação de trabalhos publicados em eventos científicos pelos experts da Petrobras, que discutiram especificamente a atuação deles na avaliação ambiental do Projeto Mexilhão.

Paralelamente, foram realizadas e gravadas 33 entrevistas semiestruturadas com os principais atores da arena do projeto, as quais envolveram, principalmente, i) um gerente do Projeto Mexilhão da Petrobras na área de Segurança, Meio Ambiente e Saúde, dois experts do setor de Engenharia de Avaliação Ambiental (EAMB) da mesma empresa, um coordenador e responsável pelo estudo e relatório de impacto ambiental do empreendimento, dois pesquisadores do Laboratório de Riscos Ambientais do Instituto de Pesquisa Tecnológica (IPT) e um pesquisador do Departamento de Ciências Atmosféricas da Universidade de São Paulo (USP), todos contratados pela Petrobras; ii) quatro analistas ambientais da Coordenação de Energia Elétrica, Nuclear e Dutos (COEND) do Instituto Brasileiro de Meio Ambiente e Recursos Naturais Renováveis (IBAMA), envolvidos e responsáveis pelo licenciamento ambiental do Mexilhão, e um analista ambiental do Escritório Regional do IBAMA em Caraguatatuba; iii) dois peritos da área de meio ambiente do Centro de Apoio à Execução (CAEX) do MPSP e dois peritos do Ministério Público Federal; iv) três gestores de unidades de conservação do LN ligados ao Instituto Florestal (IF) do Estado de São Paulo; v) um pesquisador do Instituto Tecnológico da Aeronáutica (ITA) e membro de ONG ambientalista da região do Vale do Paraíba; vi) um pesquisador do Instituto de Botânica do Estado de São Paulo; vii) três secretários municipais de meio ambiente do LN; viii) dez membros 
de organizações e associações não governamentais ambientalistas do LN.

Essas entrevistas totalizaram mais de 60 horas de gravação e foram realizadas presencialmente em diferentes municípios do LN de São Paulo, São José dos Campos, Taubaté, São Paulo, Rio de Janeiro e Brasília. O período de coleta desses dados foi de março de 2009 até novembro de 2014.

Os dados documentais e de entrevista passaram por análise e descrição objetiva, sistemática e qualitativa do conteúdo (Bardin, 1977). A análise de conteúdo desse material focou nos modos de participação, envolvimento e posicionamento desses atores no processo de licenciamento ambiental.

\section{Conflitos entre a exploração de petróleo e gás e a conservação da Mata Atlântica}

O desenvolvimento de infraestrutura de energia e de transportes tem sido um dos principais fatores para a conversão de ecossistemas naturais no Brasil desde o século XIX. No caso do setor petrolífero, apesar de a maior parte da produção de petróleo e gás estar localizada no oceano ${ }^{4}$ (offshore), as atividades de refino, tratamento, processamento e escoamento ocorrem, necessariamente, nas regiões costeiras e litorâneas. No contexto brasileiro, essa infraestrutura petrolífera tem se expandido nos últimos anos no ameaçado bioma da Mata Atlântica e em seus ecossistemas costeiros associados, em razão da descoberta e exploração das reservas do Pré-sal.

A Mata Atlântica é considerada um dos hotspot mundiais da biodiversidade por ser uma das áreas mais ricas em biodiversidade e endemismos no planeta e, ao mesmo tempo, uma das mais ameaçadas e com maior perda de habitat ${ }^{5}$ (Myers et al., 2000; Galindo-Leal \& Câmara, 2003). É a segunda maior floresta tropical do continente americano e originalmente ocupava uma área de 148,194,638 ha ao longo da costa brasileira ( $92 \%$ da costa), penetrando até o leste do Paraguai e o nordeste da Argentina, na sua porção sul (Galindo-Leal \& Câmara, 2003; Ribeiro et al., 2009). Atualmente, somados todos os fragmentos de floresta nativa acima de três hectares, restam apenas $12,5 \%$ da vegetação original no território brasileiro (Fundação SOS Mata Atlântica e Inpe, 2015), e apenas 14,4\% desse remanescente está legalmente protegido (Ribeiro et al., 2009).

Segundo Ferreira (1996), a Serra do Mar tem sido palco de inúmeros tipos de conflitos relacionados aos diferentes usos de recursos naturais durante a história de ocupação do litoral paulista. Além do setor de petróleo e gás, outros usos desafiam a gestão dos ecossistemas costeiros do bioma da Mata Atlântica brasileira: a urbanização, as atividades portuárias e de construção naval, a agropecuária e o próprio turismo (Viglio, 2012; Teixeira, 2013; Ferreira et al., 2017). No entanto, o foco deste trabalho, relacionado aos conflitos entre conservação da biodiversidade e o setor petrolífero, justifica-se

\footnotetext{
${ }^{4}$ Segundo dados da Agência Nacional de Petróleo, Gás e Biocombustíveis (ANP, 2011), em 2010, no Brasil, 94\% dos 14,2 bilhões de barris de reservas nacionais provadas de petróleo localizavam-se no mar (campos offshore). No caso do gás natural, a produção offshore correspondeu, em 2015, a 76,1\% do total produzido no país, totalizando 26,7 bilhões de $\mathrm{m}^{3}$ (ANP, 2015).

${ }^{5}$ Com base no censo populacional de 2010 do Instituto Brasileiro de Geografia e Estatística (IBGE, 2010), vivem no domínio da Mata Atlântica cerca de 112 milhões de habitantes, mais de $61 \%$ da população do Brasil. As atividades econômicas desenvolvidas nesse domínio representam aproximadamente $60 \%$ do Produto Interno Bruto (PIB) nacional.
} 
não somente pela sobreposição espacial desses diferentes usos e interesses, mas também pelas características e especificidades desse setor, as quais desafiam a política de conservação no país. Sob a ótica nacional brasileira, o setor de petróleo e gás representa i) um dos setores economicamente mais importantes e estratégicos, tecnologicamente avançados e propulsores de mudanças na estrutura populacional, assim como no emprego, na malha urbana, no quadro político e na cultura local (Egler, 1996; Piquet \& Serra, 2007; Piquet, 2012; Viglio, 2012); ii) um setor com grande legitimidade política e social, na medida em que a garantia da propriedade das jazidas petrolíferas pela União, por meio da presença da Petrobras, e o domínio da tecnologia de exploração marítima sempre foram vistos como poderosos e estratégicos instrumentos a serviço do desenvolvimento nacional (Piquet, 2012); iii) o setor energético que receberá mais investimentos públicos e privados na próxima década, segundo o Plano Decenal de Expansão de Energia, o qual indica que, entre 2013 e 2022, serão investidos 1,15 trilhões de reais no setor energético e, desse total, $72,5 \%$ irão para o setor de petróleo e gás (Ministério de Minas e Energia, 2013); iv) as obras desse setor são passíveis de serem definidas como de utilidade pública, o que lhe assegura a possibilidade de intervenção em Áreas de Preservação Permanente (APPs) e em Unidades de Conservação (UCs) de proteção integral, segundo o disposto na lei ${ }^{6}$.

A literatura que trata do conflito entre petróleo e Mata Atlântica no Brasil ainda é escassa, principalmente para o contexto do Estado de São Paulo. Alguns trabalhos recentes analisaram esses conflitos no Estado do Rio de Janeiro, especificamente o conflito que envolve a implantação do Complexo Petroquímico do Rio de Janeiro (COMPERJ), da Petrobras, em suas interfaces com as unidades de conservação da natureza componentes do Mosaico da Mata Atlântica Central Fluminense (MCF) ${ }^{7}$ (Giulianni, 2007; Dias et al., 2013; Rougemont \& Peréz, 2013; Coelho et al., 2015). Coelho et al. (2015) concluem que uma posição em conjunto das unidades de conservação, associada à ampliação da participação social das populações diretamente envolvidas, tem necessariamente mais magnitude e importância que posicionamentos particulares e isolados. Os autores defendem e entendem o papel efetivo dos mosaicos nas negociações com o setor petrolífero.

Por outro lado, Giulianni (2007) apontou a existência de contradições entre os próprios conselhos gestores das UCs como um fator complicador nas negociações. Mesmo desaprovando o empreendimento devido às consequências negativas para a conservação na região, este autor indica que as UCs têm pouca influência nas decisões do setor petrolífero e no Programa de Aceleração do Crescimento

\footnotetext{
${ }^{6}$ De acordo com a Resolução n. 369/2006 do Conselho Nacional do Meio Ambiente CONAMA, que regula o Código Florestal, instituído pela Lei n. 4.771/1965, ficou estabelecido que, em casos de interesse social e utilidade pública, é possível a intervenção ou supressão de vegetação em Área de Preservação Permanente. A intervenção em Unidade de Conservação está prevista no SNUC (Lei n. 9.985/2000), no § 3o: “Quando o empreendimento afetar unidade de conservação específica ou sua zona de amortecimento, o licenciamento a que se refere o caput deste artigo só poderá ser concedido mediante autorização do órgão responsável por sua administração, e a unidade afetada, mesmo que não pertencente ao Grupo de Proteção Integral, deverá ser uma das beneficiárias da compensação definida neste artigo".

7 Mosaico é, então, um instrumento que estimula a gestão integrada das áreas protegidas, de maneira a potencializar os esforços e otimizar o empenho dos recursos para fortalecer essas áreas e ampliar os objetivos de conservação para o território abrangido por elas. O Mosaico da Mata Atlântica Central Fluminense (MCF) foi oficialmente reconhecido pela Portaria do Ministério do Meio Ambiente n. 350/2006 (Costa et al., 2010).
} 
(PAC), no qual o empreendimento foi inserido. Além disso, para Dias et al. (2013), os investimentos privados, mediante medidas mitigadoras, compensatórias e de ajustamento de conduta, vêm ampliando cada vez mais a influência das empresas sobre o Estado e os órgãos ambientais, reduzindo e constrangendo as ações de licenciamento ambiental, de fiscalização, de monitoramento e de controle dos bens comuns, por meio de conflitos de interesses.

Em um âmbito mais geral, Reid \& Souza (2005) entendem que a melhor relação entre conservação ambiental e obras de infraestrutura e energia depende mais de sua integração no planejamento que do investimento no processo de avaliação ambiental de projetos isolados, que teria se mostrado altamente ineficaz, na perspectiva dos autores. Nessa mesma linha, Garcia (2007) afirma que, no setor de petróleo e gás no Brasil, os riscos à biodiversidade marinha e costeira não têm sido incorporados aos níveis mais estratégicos (Políticas, Planos e Programas - PPP), o que na perspectiva da autora contribui para a recorrência de acidentes e danos com consequências severas e catastróficas para a biodiversidade.

\section{O Projeto Mexilhão e o Litoral Norte do Estado de São Paulo}

O Projeto Mexilhão foi anunciado em 2005 e contou com investimento próximo aos dois bilhões de dólares. Com o objetivo de produzir cerca de 15 milhões de $\mathrm{m}^{3}$ de gás por dia, o empreendimento envolveu a instalação de uma plataforma marinha fixa e um duto submarino de $145 \mathrm{~km}$ (parte marinha) ligando essa plataforma à unidade de tratamento de gás em Caraguatatuba/SP (UTGCA). A UTGCA tem como objetivo processar o gás e o condensado, provindos dos campos de Mexilhão e Lula. Após processamento na UTGCA, o gás é transportado pelo gasoduto Caraguatatuba-Taubaté (GASTAU) de $94 \mathrm{~km}$ de extensão até o município de Taubaté, no Estado de São Paulo. O licenciamento ambiental do projeto Mexilhão foi dividido pelo IBAMA em três processos separados, envolvendo a parte marinha, UTGCA e GASTAU.

O empreendimento esteve inserido em macro programas e planos governamentais de crescimento econômico e autossuficiência energética, como o Programa de Aceleração do Crescimento (PAC) e o Plano Nacional de Antecipação da Produção de Gás (Plangás), ambos ligados ao governo federal. Além disso, o projeto foi categorizado como de interesse público, designação que permite a intervenção direta em UCs de proteção integral, como o PESM, e demais APPs.

A região costeira definida para a implantação de parte do empreendimento, o litoral norte (LN) do Estado de São Paulo ${ }^{8}$, tem sido lócus de intensas transformações demográficas e territoriais nas últimas décadas, passando de uma região de vilarejos tradicionais para uma região urbanizada. Esse processo é decorrente principalmente do desenvolvimento da atividade turística - que teve início na década de 1950 e persiste até a atualidade (Abud, 2010) -, da implantação de grandes obras de infraestrutura - como o terminal de óleo Almirante Barroso (TEBAR), o Porto de São Sebastião

\footnotetext{
${ }^{8}$ Segundo o Censo do Instituto Brasileiro de Geografia e Estatística (IBGE) de 2010, é constituído pelos municípios de São Sebastião (73.833 habitantes), Ilhabela (28.196 habitantes) e Ubatuba (78.801 habitantes).
} 
e diversas rodovias, como a construção da Rodovia dos Tamoios -, e especialmente da construção e do asfaltamento da Rodovia Rio-Santos, que estabeleceu uma ligação perene, por terra, de todo o LN.

No LN tem-se observado um dos mais acentuados processos de expansão e crescimento urbano do Estado de São Paulo. Seus municípios tiveram, entre 2000-2010, um período de crescimento urbano e econômico decorrente da instalação de empreendimentos de exploração de gás e petróleo e da consolidação de um novo tipo de turismo, assim como da própria urbanização (Marandola et al., 2013).

Nessa região, observa-se uma marcada e forte politização e institucionalização das questões de conservação e controle ambientais (Teixeira, 2013; Ferreira et al., 2011). O LN possui uma densa rede de organizações da sociedade civil em torno das questões ambientais, a qual reúne cerca de 124 ONGs ambientalistas (Souza, 2007). Estão presentes também diversas instâncias participativas e deliberativas, como conselhos municipais de meio ambiente, Comitê de Bacia Hidrográfica do Litoral Norte, Grupo Setorial de Gerenciamento Costeiro, fóruns da Agenda 21 e conselhos nas diferentes categorias das unidades de conservação.

Além de todos os municípios do LN possuírem Secretaria Municipal de Meio Ambiente, estão presentes na região representantes dos principais órgãos de controle, conservação e fiscalização ambiental do país: Instituto Brasileiro do Meio Ambiente e dos Recursos Naturais Renováveis (IBAMA), Instituto Chico Mendes de Conservação da Biodiversidade (ICMBIO), Companhia Ambiental do Estado de São Paulo (CETESB) e Instituto
Florestal do Estado de São Paulo (Ferreira et al., 2011). O MPSP tem constante atuação na região por meio do Grupo Especial para a Defesa do Meio Ambiente (GAEMA).

No que se refere à produção de conhecimento científico relacionado às questões ambientais, o LN de São Paulo é alvo de inúmeras pesquisas. Merecem destaque os centros de pesquisa da Universidade de São Paulo (USP), como o Centro de Biologia Marinha (CEBIMar), em São Sebastião, e o Instituto Oceanográfico (IO), no município de Ubatuba. São ainda desenvolvidos no LN inúmeros projetos de pesquisa vinculados aos grandes programas da Fundação de Amparo à Pesquisa do Estado de São Paulo (FAPESP), como o programa de pesquisa em Caracterização, Conservação, Restauração e Uso Sustentável da Biodiversidade (BIOTA-FAPESP) ${ }^{9}$ (Joly \& Speglich, 2003; 2005; FAPESP, 2008; Joly et al., 2011) e o Programa FAPESP de Pesquisa sobre Mudanças Climáticas Globais - $\mathrm{PFPMCG}^{10}$.

Esse dinamismo sociopolítico e científico em torno das questões ambientais no $\mathrm{LN}$ pode ser atribuído à existência de um dos maiores remanescentes de Mata Atlântica do PESM. Segundo Ribeiro et al., (2009), devido ao fato de manter $36,5 \%$ de sua vegetação original, a Serra do Mar se constitui tanto na porção mais conservada quanto no maior fragmento remanescente da Mata Atlântica. Esse fragmento, localizado principalmente ao longo das montanhas costeiras do Estado de São Paulo, possui 1.109.546 ha de floresta contínua, representando $7 \%$ do que ainda resta. Além disso, 25,2\% dessa floresta está sob proteção, majoritariamente no PESM (Ribeiro et al., 2009).

\footnotetext{
${ }^{9}$ Disponível em: <http://www.biota.org.br/>. Acesso em: 18 out. 2017.

${ }^{10}$ Disponível em: <http://www.fapesp.br/pfpmcg/>. Acesso em: 18 out. 2017.
} 
O PESM, gerenciado pelo Instituto Florestal do Estado de São Paulo, é a maior UC de proteção integral do litoral brasileiro, com 315.390 ha, abrangendo 23 municípios. Foi criado em 1977 com a finalidade de assegurar a proteção integral da flora, da fauna, das belezas naturais, bem como para garantir sua utilização com objetivos educacionais, recreativos e científicos (SMA, 2006). Esse parque contém as maiores porções dos rios que deságuam no Atlântico e abastecem os centros urbanos costeiros (Evans, 2007). Além disso, devido à sua localização, o PESM constitui um verdadeiro corredor ecológico, conectando os mais significativos remanescentes de Mata Atlântica do país.

A área do PESM apresenta ainda características histórico-culturais valiosas, mantidas pelas comunidades tradicionais que registram os diversos momentos da ocupação humana na Serra do Mar (Calvimontes, 2013; Simões, 2010). Por todos esses motivos, o PESM é hoje um polo de concentração das atenções de toda a comunidade científica, governos, empresas privadas e demais setores da sociedade, em razão da preocupação com a preservação da Mata Atlântica e da necessidade de aprofundamento dos conhecimentos sobre a fauna e a flora regionais.

Em suma, o LN de São Paulo reúne um conjunto de fatores ambientais e sociopolíticos que influenciaram nas negociações em torno do Projeto Mexilhão, como elementos bióticos, físicos e paisagísticos de forte apelo conservacionista, forte politização regional em relação às questões ambientais, marcada institucionalização de instâncias democráticas de decisão e vasta produção científica sobre as questões ambientais.

\section{Resultados e discussão}

\subsection{A arena do licenciamento ambiental do Projeto Mexilhão}

Em geral, os atores em disputa na arena do licenciamento ambiental do Projeto Mexilhão podem ser descritos em um continuum de posições favoráveis e críticas ao empreendimento. Em um dos extremos, encontravam-se a Petrobras e o Governo Federal, para os quais a obra representava o elo fundamental que faltava para tornar o Brasil autossuficiente em gás natural. Eles tiveram o apoio principalmente dos prefeitos dos municípios do litoral paulista, que disputaram a instalação da unidade de tratamento de gás nessas localidades (Francine Junior, 2012). Na ocasião, havia previsão da geração de cerca de 19 mil empregos, não fixos. Quando em funcionamento, o projeto previa a geração de aproximadamente 800 empregos diretos e de 6 mil a 7 mil indiretos. Além disso, havia previsão de aumento de arrecadação das receitas municipais - no caso de Caraguatatuba, o principal município envolvido, o aumento viria com royalties e impostos, como o Imposto sobre Operações sobre a Circulação de Mercadorias e Serviço (ICMS) e o Imposto sobre Serviço de Qualquer Natureza (ISSQN). Era esperado um acréscimo de R\$ 150 milhões no orçamento municipal. Tudo isso em um contexto em que Caraguatatuba exibia, segundo o censo do IBGE de 2000, indicadores sociais abaixo da média paulista, com renda média per capita de 2,16 mínimos contra 2,92 do Estado. Além disso, $20 \%$ das casas não apresentavam infraestrutura urbana adequada - no Estado, o índice era de 10,7\% 
(Jornal Folha de São Paulo, 2007).

No outro extremo, havia uma crítica explícita ao Projeto Mexilhão realizada por membros de ONGs ambientalistas do LN e do Vale do Paraíba, pesquisadores do Instituto de Pesquisa Tecnológica (IPT), peritos e promotores do Ministério Público do Estado de São Paulo. O Mexilhão representava para esses atores um empreendimento potencialmente poluente e capaz de afetar a conservação ambiental e o turismo. Esses atores também criticaram a divisão do licenciamento ambiental em três processos compartimentados feita pelo IBAMA. Isso dificultava, na perspectiva deles, a avaliação dos impactos cumulativos e sinérgicos das partes do empreendimento e deste com outras intervenções que estavam sendo instaladas e planejadas no LN de São Paulo naquela ocasião.

Já as posições intermediárias reconheciam os potenciais riscos e impactos do empreendimento, reconheciam benefícios sociais e econômicos e interpretaram o empreendimento como inevitável por suas credenciais políticas e econômicas. Dessa forma, resolveram concentrar seus esforços na definição e na implementação de medidas mitigadoras e compensatórias. Nessa área do espectro de posições encontravam-se, sobretudo, a maior parte das ONGs ambientalistas do LN, peritos do Ministério Público Federal e do IBAMA, gestores de UCs diretamente afetadas pelo empreendimento e outros membros da Secretaria de Meio Ambiente do Estado de São Paulo.

\subsection{Disputas e negociações sobre as implicações ambientais do Projeto Mexilhão no Parque Estadual da Serra do Mar}

Os conflitos envolvendo o Projeto Mexilhão decorreram da decisão da Petrobras em instalar a unidade de tratamento de gás no município de $\mathrm{Ca}$ raguatatuba. Essa planta industrial foi construída a apenas $1 \mathrm{~km}$ de distância do PESM e dentro de sua zona de amortecimento1111. Consequentemente, o gasoduto (GASTAU) para o transporte de gás processado desde essa planta até a conexão com o gasoduto Campinas-Rio de Janeiro, em Taubaté, deveria atravessar o PESM ao longo de 5,2 km. Esse conflito envolveu, sobretudo, a Petrobras, o IBAMA, ONGs ambientalistas, o Ministério Público e a Secretaria Estadual de Meio Ambiente de São Paulo.

Para os analistas do IBAMA entrevistados, juntamente com os pesquisadores do ITA e peritos do MPSP, o município de Caraguatatuba não era a escolha mais viável, do ponto de vista ambiental, para a instalação do empreendimento. Esses analistas apontaram como problemáticas tanto a proximidade da UTGCA com o PESM quanto a intervenção direta do GASTAU. Entretanto, os analistas ambientais não acreditavam ou não se viam com autonomia e respaldo político para impedir ou negar as licenças ambientais do empreendimento, visto que se tratava de um projeto vinculado à Petrobras e inserido no PAC do Governo Federal. O depoimento do ex-analista ambiental responsável pelos processos de licenciamento da UTGCA e

11 A Zona de Amortecimento (ZA, também chamada de "Zona Tampão") é uma área estabelecida ao redor de uma unidade de conservação. Ela foi criada pelo artigo $2^{\circ}$, inciso XVIII da Lei do SNUC (Lei n. 9.985/2000), que a define como o "entorno de uma unidade de conservação, onde as atividades humanas estão sujeitas a normas e restrições específicas, com o propósito de minimizar os impactos negativos sobre a unidade". 
do GASTAU evidenciou esse posicionamento do seguinte modo:

Um empreendimento que veio ao IBAMA para ser licenciado como uma obra que seria um avanço para o desenvolvimento do país, a gente sabia que não inviabilizaria, independente do tempo que levasse. Não era um empreendimento que seria inviabilizado, mesmo com todos os problemas ambientais (Entrevista com ex-analista ambiental da Dilic/IBAMA, maio de 2011).

Essa constatação de restrições e limites no âmbito do principal órgão de regulação ambiental do país vai de encontro com os achados de outros trabalhos, como o de Piagentini \& Favareto (2014) e de Dias et al. (2013). Estes últimos, por exemplo, sustentam que os órgãos ambientais vêm tendo suas atribuições gradualmente restringidas com a existência de constrangimentos internos aos técnicos que apresentam argumentos contrários à forma como se dá a implantação dos empreendimentos. Isso sugere também que as decisões sobre a localização de empreendimentos como o Mexilhão estão em jogo e se dão em outras arenas, como a do planejamento energético, que não contam com o envolvimento e a participação dos analistas ambientais do IBAMA.

No caso do Projeto Mexilhão, este trabalho constatou que, diante dessas restrições, os analistas ambientais do IBAMA envolvidos no licenciamento buscaram dissuadir a Petrobras da construção da UTGCA e do GASTAU na região por ela definida. Segundo o ex-analista do IBAMA responsável pelo licenciamento do projeto, a recomendação para a construção de um túnel em rocha para a transposição do GASTAU no interior do PESM, que foi colocada como uma condição pelo IBAMA para a concessão das licenças ambientais, visava inicialmente que a Petrobras desistisse de construir o empreendimento naquela localidade. Na concepção deste entrevistado, o alto custo e a complexidade técnica do túnel poderia fazer com que a Petrobras procurasse alternativas locacionais. No entanto, essa estratégia não surtiu o efeito esperado pelo IBAMA, pois a Petrobras aceitou a proposta de construção do túnel, mas apenas dentro do limite do PESM. O trecho abaixo, extraído de entrevista com o então responsável pelo licenciamento ambiental da UTGCA e do GASTAU, é elucidativo desta interpretação:

Esse túnel foi uma proposta inicial que o IBAMA jamais imaginou que a Petrobras realizaria, pois a gente sabia das dificuldades. A intenção do IBAMA era fazer com que o empreendimento avaliasse outras áreas menos impactantes. Mas foi feita uma série de estudos do túnel pela empresa e ele se constituiu como uma alternativa viável para minimizar os impactos sobre o parque e que acabou sendo aceita pela Fundação Florestal, que era gestora do parque, que participou o tempo todo dessa negociação e a gente chegou a esse consenso (Entrevista com ex-analista ambiental do IBAMA, responsável pelo licenciamento da UTGCA e GASTAU, em abril de 2011).

O processo de negociação que ocorreu antes da realização dos EIAs do empreendimento e que levou a decisão da Petrobras em construir um túnel para o GASTAU na área do PESM contou também com a participação de membros do Instituto Florestal do Estado de São Paulo, incluindo o gestor do Núcleo Caraguatatuba do PESM. A proposta original da Petrobras envolvia a construção do gasoduto pela superfície do PESM. Essa proposta acarretaria uma intervenção direta no parque devido à supressão de vegetação para a construção do gasoduto e de sua faixa de servidão. Essa posição pode ser evidenciada 
na fala do gerente do Projeto Mexilhão, entrevistado neste trabalho:

O gasoduto também foi complicado, pois tinha que atravessar a Serra do Mar porque, no início do projeto, o gasoduto seria atravessando a serra, desmatando a serra. Mas os órgãos ambientais não aceitaram de maneira nenhuma isso, por diversas razões ambientais, que não vou entrar no mérito disso, se está certo ou errado. Até que chegamos à conclusão que fazer um túnel seria a solução mais adequada ambientalmente falando (Entrevista com o Gerente do Empreendimento na Área de Segurança, Meio Ambiente e Saúde, março de 2011).

A proposição inicial da Petrobras de interferência direta no PESM teria respaldo legal devido à classificação do empreendimento como obra utilidade pública pelo governo Federal ${ }^{12}$, o que permite a flexibilização do arcabouço legal voltado à conservação ambiental. No caso específico do Estado de São Paulo, o artigo 24 do Decreto n. 25.341/1986, que aprovou o regulamento dos Parques Estaduais Paulistas, indicava que "é vedada a execução de obras que visem à construção de teleféricos, ferrovias, rodovias, barragens, aquedutos, oleodutos, linhas de transmissão ou outras, que não sejam de interesse do Parque Estadual' (São Paulo, 1986). No entanto, esse artigo foi modificado pelo Decreto Estadual n. 29.762/1989, que determinou a inclusão de um parágrafo único com os objetivos de minimizar a rigidez do Decreto n. 25.341/1986 e permitir a realização de obras consideradas de interesse público, conforme expresso:

Parágrafo único - Não se compreendem na reda- ção contida no "caput" deste artigo as obras nele referidas que sejam consideradas de utilidade, de necessidade pública ou de interesse social e que não comprometam a integridade dos atributos que justificam a proteção do Parque Estadual, atestados pelos órgãos oficiais competentes, mediante estudo prévio do impacto ambiental, a que se dará publicidade (São Paulo, 1989; destaque dos autores).

Segundo o depoimento de um perito do Ministério Público do Estado de São Paulo, com base nessa modificação, "vários dutos, estradas e outras obras passaram a ser propostos e licenciados pelos órgãos competentes, interceptando o Parque Estadual da Serra do Mar" (Entrevista por e-mail com perito do MPSP, em março de 2014). No caso do Mexilhão, mesmo que o túnel tenha se constituído inicialmente como uma estratégia para a desistência da Petrobras, o fato de o IBAMA ter conseguido colocá-lo como uma condição na negociação indica o peso político e social que tem o PESM e os atores a ele ligados no Estado São Paulo. Dessa forma, ainda que a Petrobras tivesse respaldo legal para intervenção direta no PESM, os possíveis desdobramentos dessa intervenção comprometeriam tanto a execução do empreendimento quanto a própria imagem da empresa.

Este trabalho mostra que a adoção de medidas de mitigação, como no caso do túnel, não foram aceitas e estabelecidas automaticamente sem a existência de disputas, negociações e contingências. A mitigação e a compensação ambiental, como instrumentos presentes na legislação, desde que efetivados, também podem contribuir para a conservação (Reid \& Souza, 2005) e, assim, são relevantes

\footnotetext{
${ }^{12}$ Decreto-Lei n. 3.365/1941: dispõe sobre desapropriação por utilidade pública.
} 
analiticamente no jogo das decisões sobre grandes projetos de energia e infraestrutura.

Ao mesmo tempo, é importante salientar que as características do túnel, como principal medida de mitigação, envolvendo sua dimensão e ineditismo, evidencia o montante de recursos técnicos e financeiros que a Petrobras dispõe para sustentar suas decisões. Naquele momento, esse túnel se constituía como a mais longa escavação em rocha a partir de um único emboque no Brasil. Seus 5,2 $\mathrm{km}$ de extensão e seus $6,19 \mathrm{~m}$ de diâmetro atingiram escavações de até $540 \mathrm{~m}$ de profundidade a um custo aproximado de 100 milhões de dólares (Jornal Folha de São Paulo, 9 de fevereiro de 2012). A construção do túnel do GASTAU foi utilizada pela Petrobras, nos EIAs-RIMAs e em audiências públicas, como um dos principais argumentos para indicar a sustentabilidade ambiental do Projeto Mexilhão (Figura 1).
Com base nessa decisão, a resistência em relação à construção do empreendimento foi minimizada, visto que a decisão da empresa contribuiu para maior aceitação e legitimidade do empreendimento, tanto por parte do IBAMA quanto de outros atores da arena, como as ONGs ambientalistas e os peritos do MPF.

Já as negociações e solicitações para o prolongamento do túnel por mais $3 \mathrm{~km}$ além do limite do PESM, envolvendo também o MPSP, não teve o mesmo êxito. Essa posição da Petrobras levou à redução da Mata Atlântica em aproximadamente 22 hectares. A empresa justificou, por meio de pareceres técnicos, que para a execução de um túnel de 8 $\mathrm{km}(5,2 \mathrm{~km}$ no parque e $3 \mathrm{~km}$ na zona de amortecimento) seria necessária a abertura de um poço de ventilação de grande diâmetro no interior do PESM. De acordo com a Petrobras, seriam necessários novos acessos dentro do parque para transporte de

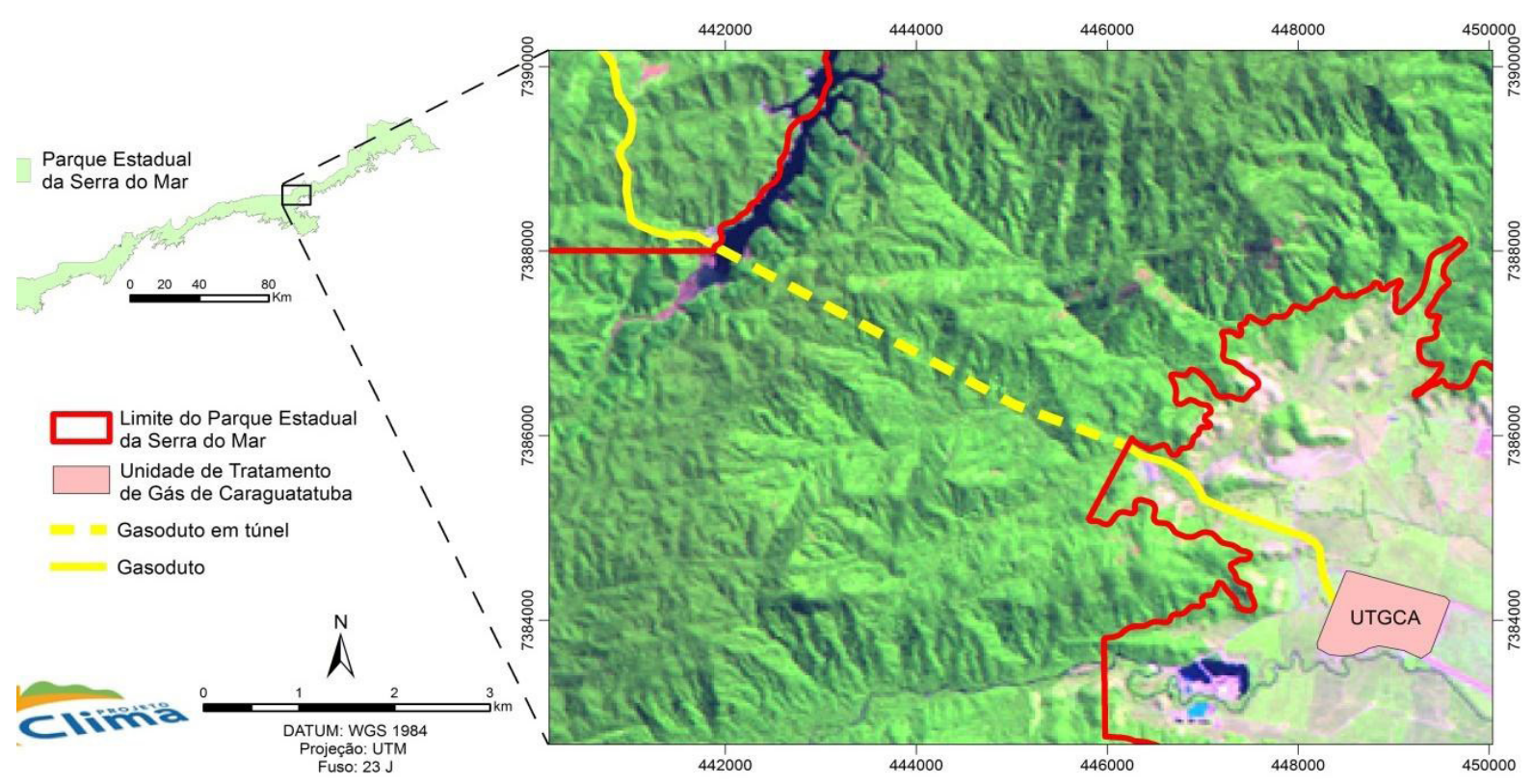

FIGURA 1 - Traçado do GASTAU e do túnel no PESM. 
maquinário, pessoas e materiais (SMS/AC/SP-CO 010/2008, respondendo ao oficio do Inquérito Civil 23/2005). Essa argumentação foi questionada pelo MPSP, que mobilizou pesquisadores do Instituto de Tecnologia da Aeronáutica (ITA) para embasar sua posição, como pode ser observado em trecho retirado de parecer do MPSP:

A nota técnica da Petrobras que versa sobre a hipótese do prolongamento do túnel ( $5 \mathrm{~km}$ para $8 \mathrm{~km}$ ), minimizando impactos em região de grande significância ambiental, não se mostrou suficiente para responder aos questionamentos levantados pelos professores colaboradores do MPSP sobre o tema. (...). Segundo os professores do ITA, a Petrobras não apresentaa quantificação e comparação sobre os riscos e as necessidades de suporte em relação a sua tese. Segundo a Petrobras, o prolongamento exigiria outro sistema de ventilação, o que necessitaria abrir um poço de ventilação de grande diâmetro no interior do PESM. Segundo ITA, existe maquinário que permite a perfuração de poços com mais de $2 \mathrm{~m}$ de diâmetro por várias centenas de metros, sendo desnecessário um poço de grande diâmetro. (Parecer Técnico CAEX RI n. 3.368, de 8 de março de 2012)

Além das divergências técnico-científicas entre os atores em oposição na arena, como o MPSP e a Petrobras, foi possível observar interpretações diferentes no âmbito do próprio Ministério Público acerca das implicações ambientais do Projeto Mexilhão. Enquanto os peritos do MPSP rejeitaram os estudos técnicos e a posição da Petrobras quanto aos danos ambientais e à insuficiência das medidas de mitigação propostas, os experts do MPF avaliaram que tais estudos, incluindo o EIA-RIMA, salvo alguns apontamentos e correções, eram adequados e atendiam aos requisitos técnicos e legais do licenciamento ambiental. Esse posicionamento dos peritos do MPF é evidenciado conforme o trecho a seguir de entrevista:

Então, veja só: a UTGCA ia ser construída numa área que tem gramínea, a faixa de dutos seria por túnel para atravessar e não interferir no Parque Estadual da Serra do Mar. Então, a gente precisa pesar os prós e os contras de forma mais isenta. A gente entende que se é um empreendimento que tem todas as chances de ser licenciado, mas com critérios ambientais, com cuidados sérios, com compensações, com medidas adequadas, a gente acha que nosso trabalho é mais produtivo com esse foco (de propor melhorias na forma de medidas mitigadoras e compensatórias) do que simplesmente a gente dizer não por não, não, não quero que Caraguatatuba tenha isso, então é não! Claro, a gente com formação na área ambiental pensamos muitas vezes, "puxa, é uma pena a ocorrência destes impactos, a questão paisagística". É claro que a gente é sensivel a isso, mas a gente tem que trabalhar com levantamentos, com dados, a gente tem que trabalhar com os pés no chão. (Entrevista com Perito do MPF, agosto 2011)

Essa divergência no âmbito do MP impediu um posicionamento único da própria instituição quanto aos impactos ambientais do Projeto Mexilhão. Tal divergência, por exemplo, foi utilizada por outros atores da arena, como o presidente do IBAMA, para sustentar sua decisão pela concessão das licenças ambientais do empreendimento e, assim, contrapor a posição do MPSP. Esse presidente destacou o posicionamento dos peritos do MPF que aprovou os estudos ambientais do Projeto Mexilhão, como pode ser evidenciado no seguinte trecho: "Por fim, o parecer do próprio Ministério Público que trata do empreendimento considera que as informações apresentadas em sua maior parte atendem ao termo de referência" (OFÍCIO 164/2008 - PRESIDÊNCIA DO IBAMA). 


\section{Considerações finais}

O LN de São Paulo e parte das regiões Sudeste, Nordeste e Sul do Brasil concentram os mais importantes remanescentes de Mata Atlântica e, ao mesmo tempo, são os maiores detentores de reservas offshore de petróleo e gás do país. Essa complexa sobreposição entre usos e interesses, a princípio tão divergentes, tem suscitado debates e conflitos sociais, como foi exemplificado neste estudo.

Esse quadro se torna ainda mais agravante para a conservação da biodiversidade quando se constata que somente cerca de $1,62 \%$ da área original de ocorrência da Mata Atlântica se encontra legalmente protegida. No caso da Serra do Mar, onde há uma parcela maior da mata remanescente sob proteção (25,2\%), a intensificação das atividades de petróleo e gás impulsionadas pelo Pré-sal acentua a tensão entre conservação da biodiversidade e grandes empreendimentos de infraestrutura e energia.

Como indicam os resultados desta pesquisa, a mera existência do PESM não foi suficiente para que a Petrobras reavaliasse a instalação do empreendimento em sua zona de amortecimento na arena do licenciamento ambiental. Apesar da posição de Bruner et al. (2001) e Ferreira et al. (2005) a respeito da efetividade que tem por si só a criação de UCs para a conservação da biodiversidade, no caso analisado, a integridade do PESM não estava garantida inicialmente diante da possibilidade real da construção do gasoduto que atravessaria o parque pela superfície. $O$ fato desse cenário não ter se concretizado devido à construção de um túnel não foi consequência apenas de seu status legal como área protegida, mas também, e principalmente, à presença e à atuação de atores ambientalistas governamentais e não governamentais na região.
A arena de negociação sobre o desenvolvimento do LN de São Paulo se caracteriza por ser altamente politizada e por ter forte apelo conservacionista. O histórico da região, que determina a presença de atores institucionais e individuais com importantes recursos de poder, influencia todos os processos de negociação relacionados ao uso dos recursos naturais. Esse contexto especial do LN se constitui em um exemplo de como as características da arena são determinantes para os processos de tomadas de decisão, especificamente em relação às medidas de mitigação, como foi o caso do túnel do GASTAU. A capacidade de mobilização de recursos dos atores ambientalistas historicamente presentes na região, a importância do PESM, a influência dos atores governamentais e o manejo da imagem institucional da Petrobras e seu poder político confluíram neste processo.

Considerando que o Projeto Mexilhão e seus impactos extravasaram o PESM, este acabou se constituindo, de algum modo, em moeda de barganha na arena. Sabendo que as maiores exigências e o apelo para a conservação se concentravam, sobretudo, dentro nos limites do parque, a Petrobras manteve sua posição em não prolongar o túnel para além desses limites, mesmo sabendo dos impactos nos demais remanescentes da floresta. Desse modo, se por um lado a atuação dos atores ambientalistas contribuiu para assegurar a integridade do PESM, por outro não foi suficiente para assegurar integridade da Mata Atlântica como um todo na região diante da instalação do empreendimento.

Do ponto de vista teórico-metodológico, este trabalho constata a relevância de análises multiatores que são, ao mesmo tempo, abertas analiticamente à pluralidade interna de cada ator e à possibilidade de contingência e negociação nos conflitos 
decorrentes de processos decisórios, principalmente na arena ambiental. Ao mesmo tempo, a perspectiva do processo decisório em multiníveis, como empregado aqui por meio do conceito de arena, foi de grande importância. As decisões pré-tomadas sobre o empreendimento em outras arenas que não a do licenciamento ambiental tiveram que passar por questionamentos e foram reavaliadas parcialmente por conta das negociações entre as partes.

Teríamos, portanto, que nos contentar com resultados subótimos quando o que se está em jogo é a integridade de um dos ecossistemas mais ameaçados do país? A nossa resposta é não. No entanto, o processo decisório multiatores como o do licenciamento ambiental do Mexilhão teve que encontrar e destacar pelo menos algumas metas comuns a serem atingidas e pactuadas. Uma vez alcançadas, inevitavelmente outros conflitos, outras negociações e outras metas virão à tona e se manifestarão.

O processo de negociação descrito neste trabalho é conclusivo no que se refere à importância da institucionalização das áreas protegidas como ferramentas de conservação, mais ainda em regiões como o LN, onde grandes empreendimentos estão em marcha. Por si só, a criação dessas áreas pode não ser suficiente, mas, sem dúvida, sua presença influencia nos processos de negociação e decisão. Essa influência pode ser maior ou menor em razão das características próprias da região onde elas se encontram. O status de conservação, a mobilização dos atores ambientalistas, a importância estratégica da região e a visibilidade dos atores regionais nos níveis superiores da arena são algumas das variáveis mais importantes.

\section{Referências}

Abud, J. P. D. Processo de ocupação, efeitos ambientais adversos e novos instrumentos de ordenação territorial no Litoral Norte de São Paulo, Brasil: Problemas e Perspectivas. Actas del XI Coloquio Internacional de Geocrítica. Buenos Aires, mayo de 2010.

ANP - Agência Nacional de Petróleo. Anuário estatístico brasileiro de petróleo, gás e biocombustíveis, 2015. Disponível em<http://www.anp.gov.br/?pg=78136em=et1=et2=e$\mathrm{t} 3=\mathrm{et} 4=\mathrm{ear}=\mathrm{eps}=\mathrm{e} 1458042805086>$. Acesso: ago. 2017.

ANP - Agência Nacional de Petróleo. Anuário estatístico brasileiro do petróleo, gás natural e biocombustiveis, 2011 (dados referentes a 2010): Rio de Janeiro, 2011.

Agra Filho, S. S. Conflitos ambientais e os instrumentos da política nacional de meio ambiente. In: Zhouri, A; Laschefski, K. Desenvolvimento e conflitos ambientais. Belo Horizonte: Editora UFMG, 2010.

Bardin, L. Análise de conteúdo. Lisboa: Edições 70, 1977.

Brasil, Ministério do Planejamento. Sobre o PAC. Disponível em: http://www.pac.gov.br/sobre-o-pac. Acesso Fev. 2016.

Bruner, A. G.; Gullison, R. E.; Rice, R. E.; Da Fonseca, G. A. Effectiveness of Parks in Protecting Tropical Biodiversity. Science, 291(5501), 125-128, 2001.

Calvimontes, J. U. Bandidos na Serra do Mar? Conflitos, Estratégias e Usos Múltiplos dos Recursos Naturais na Mata Atlântica. Campinas, Tese (Doutorado em Ambiente e Sociedade) - Núcleo de Estudos e Pesquisas Ambientais, Unicamp, 2013.

Carter, A. S. Extractive Industries as a New Constituency for Protected Areas. In: McNeely, J. (Ed.). Friends for Life: New Partners in Support of Protected Areas. Gland, Switzerland: IUCN, 2005.

Chow, J.; Kopp, R. J.; Portney, P. R. Energy Resource and Global Development. Science, 302, 1528-1531, 2003.

Coelho, B. H.S.; Loureiro, C. F.; Irving, M. A.; Soares, D. G. Conflitos entre o Comperj e a gestão de áreas protegidas: O Mosaico Central Fluminense como possibilidade 
de enfrentamento a impactos socioambientais de grandes empreendimentos industriais. Desenvolvimento e Meio Ambiente, 35, 259-273, 2015.

Committee on the Effects of the Deepwater Horizon Mississippi Canyon-252 Oil Spill on Ecosystem Services in the Gulf of Mexico. An Ecosystem Services Approach to Assessing the Impacts of the Deepwater Horizon Oil Spill in the Gulf of Mexico, 2013. Disponível em: http://www. nap.edu/catalog/18387/an-ecosystem-services-approach-to-assessing-the-impacts-of-the-deepwater-horizon-oil-spill-in-the-gulf-of-mexico. Acesso. Jan. 2015.

Conservation International. Energy and Biodiversity Initiative: Integrating Biodiversity Conservation into Oil and Gas Development. Energy and Biodiversity Initiative, Washington DC, USA. 2003. Disponível em: www.theebi. org/pdfs/ebi_report.pdf. Acesso: jan. 2015.

Copeland, H. E.; Doherty, K. E.; Naugle, D. E.; Pocewicz, A.; Kiesecker, J. M. Mapping Oil and Gas Development Potential in the US Intermountain West and Estimating Impacts to Species. PLoS ONE, 4(10), 2009.

Costa, C.; Lamas, I.; Fernandes, R. Planejamento estratégico do Mosaico Central Fluminense. Silva Jardim: Associação Mico-Leão-Dourado, Valor Natural, Conservação Internacional \& Fundação SOS Mata Atlântica, 2010.

Dias, A.; Souza, A.; Maia, A.; Berzins, A. Complexo Petroquímico do Rio de Janeiro (Comperj): impactos socioambientais, violação de direitos e conflitos na baía de Guanabara. Revista Ética e Filosofia Política, 1 (16), 151-175, 2013.

Dudley, N. (Ed.). Guidelines for Applying Protected Area Management Categories. Gland, Switzerland: IUCN, 2008.

Egler, C. A. G. Risco Ambiental como Critério de Gestão do Território. Revista Território, 1, 1-41, 1996.

Energy and Biodiversity Initiative. Integrating Biodiversity Conservation into Oil and Gas Development: Washington DC, USA. 2003. Disponível em www.theebi.org/pdfs/ ebi_report.pdf. Acesso: Jan. 2015.

Evans, Y. Participação Comunitária em Gestão Ambiental: o caso do Parque Estadual da Serra do Mar.

Fapesp - Fundação de Amparo à Pesquisa do Estado de
São Paulo - Knowledge and sustainable use of Brazilian biodiversity. São Paulo: FAPESP, 2008.

Ferreira, L. da C. Os Ambientalistas, os Direitos Sociais e o Universo da Cidadania. In: Ferreira, L. da C.; Viola, E. (Org.). Incertezas de Sustentabilidade na Globalização. Campinas: Editora da UNICAMP, 1996.

Ferreira, L. da C. Conflitos sociais e uso dos recursos naturais: breves comentários sobre modelos teóricos e linhas de pesquisa. Política e Sociedade, 7, 2005.

Ferreira, L. da C. A Equação Dinâmica entre Conflitos Sociais, Recursos Naturais e Desastres Ambientais: O Estado da Arte e uma Proposta Teórica. In: Anasi do VI Encontro Nacional da ANPPAS, Setembro de 2012, Belém - PA - Brasil.

Ferreira, J.; Aragão, L. E. O. C.; Barlow, J.; Barreto, P.; Berenguer, E.; Bustamante, M.; Pardini, R. Brazil's environmental leadership at risk. Science, 346(6210), 706-707, 2014.

Ferreira, L. da C.; Martins, R. D. A.; Barbi, F.; da Costa Ferreira, L.; de Mello, L. F.; Urbinatti, A. M.; Andrade, T. H. N. Governing Climate Change in Brazilian Coastal Cities: Risks and Strategies. Journal of US-China Public Administration, 10, 51-65, 2011.

Ferreira, L. V.; Venticinque, E.; Almeida, S. O desmatamento na Amazônia e a importância das áreas protegidas. Estudos Avançados, 19(53), 2005.

Ferreira, L. da C. Calvimontes, J.; Di Giulio, G.; Viglio, J. E.; Araos, F.; Feital, M. Conflictos entre expansión urbana y cobertura vegetal y sus consecuencias para los cambios ambientales globales: un estudio en el litoral del Estado de São Paulo, Brasil. In: Ferreira, L. C.; Pardo, M.; Schimitd, 1.; Calvimontes, J.; Viglio, J. E. Clima de tensão: ação humana, biodiversidade e mudanças climáticas. Campinas, SP: Editora da Unicamp, 2017.

Finer, M.; Jenkins, C. N.; Pimm, S. L.; Keane, B.; Ross, C. Oil and Gas Projects in the Western Amazon: Threats to Wilderness, Biodiversity, and Indigenous Peoples. PLoS ONE, 3(8), 2008.

Finer, M.; Orta Martinez, M. A second hydrocarbon boom threatens the Peruvian Amazon: trends, projections, and 
policy implications. Environmental Research Letters, 5,1-10, 2010.

Francine Junior, R. O Diálogo como estratégia de mediação de conflitos ambientais: Análise de aspectos do Comitê de Promoção do Diálogo para a Sustentabilidade do Litoral Norte do Estado de São Paulo COMDIAL. Belo Horizonte, Trabalho de Conclusão de Curso de Pós Graduação, Núcleo de Educação a Distância, da Universidade Federal de Minas Gerais - UFMG, 2012.

Fundação Getúlio Vargas. Exploração e produção de petróleo em águas ultraprofundas: nova fronteira para o pós-crise, 2011. Disponível em: http://fgvProjetos.fgv.br/ publicacoes/exploracao-e-producao-de-petroleo-em-aguas-ultraprofundas. Acesso: Jan. 2013.

Fundação Fundação SOS Mata Atlântica \& Inpe 2013. Relatório Anual 2012, 2013. Disponível em https://www. sosma.org.br/wp-content/uploads/2013/05/relatorio-SOSMataAtlantica-2012-site.pdf. Acesso: Jan. 2014.

Fundação Fundação SOS Mata Atlântica \& Inpe. Atlas dos Remanescentes Florestais da Mata Atlântica 2013-2014. 2015. Disponível em: http://mapas.sosma.org.br/site_media/ download/atlas_2013-2014_relatorio_tecnico_2015.pdf. Acesso: Jan. 2015.

Galindo-Leal, C.; Câmara, I. G. Atlantic Forest hotspot status: an overview. In: Galindo-Leal, C.; Câmara, I. G. (Eds.). The Atlantic Forest of South America: biodiversity status, trends, and outlook. Washington: Center for Applied Biodiversity Science and Island Press, 2003.

Garcia, K. C. Avaliação Estratégica do Risco à Biodiversidade nos Planos de Programas de E\&Poffshore de Petróleo e Gás Natural no Brasil. Rio de Janeiro, (Tese) Doutorado: PPE/COPPE/UFRJ, 2007.

Giuliani, G. M. As áreas naturais protegidas e a responsabilidade social e ambiental das empresas: o caso do Mosaico da Mata Atlântica Central Fluminense e do Comperj. Desenvolvimento e Meio Ambiente, 16, 21-37, 2007.

IBGE - Instituto Brasileiro de Geografia e Estatística. Projeções Preliminares: Censo demográfico de 2010. Disponível em: http://censo2010.ibge.gov.br/ Acesso: Jan. 2014.

IUCN - The World Conservation Union. Natural world heritage sites off limits for mining and oil exploration, 2008. Disponível em: http://www.iucn.org/fr/nouvelles_homepage/nouvelles_par_theme/affaires_news/?1238/Put-natural-World-Heritage-sites-off-limits-for-mining-and-oil-exploration-IUCN-says. 2008. Acesso: fev. 2014.

Hannigan, J. Environmental sociology. Routledge, 2ed, 2006.

Hochstetler, K. The Politics of Environmental Licensing: Energy Projects of the Past and Future in Brazil. Studies in Comparative International Development, 46(4), 349-371, 2011.

Jaffe, A.; Victor, D. The Geopolitics of Natural Gas, Executive Summary. Disponível em http://pesd.fsi.stanford.edu/ sites/default/files/WP36\%2C_Conclusions.pdf. Acesso: Jan. 2014.

Joly, C. A.; Haddad, C. F.; Verdade, L. M.; Oliveira, M. C. D.; Bolzani, V. D. S.; Berlinck, R. G. Diagnóstico da pesquisa em biodiversidade no Brasil. Revista USP, 89, 114-133, 2011.

Joly, C. A.; Speglich, E. Programa BIOTA/FAPESP: um novo paradigma no estudo da conservação e do uso sustentável da biodiversidade. Ciência e Cultura ,55 (3), 41-43, 2003.

Joly, C. A.; Speglich, E. Programa BIOTA/FAPESP: um modelo brasileiro para programas de pesquisa em conservação e uso sustentável da biodiversidade. Boletín de la Sociedad Argentina de Botánica, 39, 303-306, 2005.

Jones, C. M.; Chaves, H. A. F. Assessment of yet-to-find-oil in the Pre-Salt area of Brazil. In: 14th International Congress of the Brazilian Geophysical Society \& EXPOGEF. Rio de Janeiro, 3-6 August, 2015.

Jornal Folha de São Paulo. À beira da rodovia, bairro concentra migrantes. Caderno Cotidiano. 18 de novembro de 2007. Disponível em http://www1.folha.uol.com.br/fsp/ cotidian/ff1811200707.htm. Acesso: Jun. 2012.

Jornal Folha de São Paulo. Petrobras enterrou máquina de $R \$ 51$ milhões. Mercado, 9 de Fevereiro de 2012. Disponível em: http://www1.folha.uol.com.br/fsp/mercado/24850-petrobras-enterrou-maquina-de-r-51-mi.shtml. Acesso: Jul. 2015. 
Lessmann, J.; Fajardo, J.; Munoz, J.; Bonaccorso, E. Large expansion of oil industry in the Ecuadorian Amazon: biodiversity vulnerability and conservation alternatives. Ecology and evolution, 6(14), 4997-5012, 2016.

Leyen, B. C. Eco-eficiência na exploração e produção de petróleo e gás em regiões de florestas tropicais úmidas: o caso da Petrobrás na Amazônia. Rio de janeiro, Dissertação (Mestrado), COPPE/UFRJ, 2008.

Marandola Jr, E.; Marques, C.; Paula, L. T. D.; Cassaneli, L. B. Crescimento urbano e áreas de risco no litoral norte de São Paulo. Revista Brasileira de Estudos de População, 30, 35-56, 2013.

Maugeri, L. Oil: The Next Revolution. Discussion Paper, Belfer Center for Science and International Affairs, Harvard Kennedy School, 2012.

Ministério de Minas e Energia. Secretaria de Planejamento e Desenvolvimento Energético. Plano Decenal de Expansão de Energia-PDE 2022, 2013. Disponível em: http://www. epe.gov.br/PDEE/20140124_1.pdf. Acesso: Jan. 2015.

Miranda, M.; Burris, P.; Bingcang, J. F.; Shearman, P.; Briones, J. O.; La Vinã, A.; Menard, S. Mining and Critical Ecosystems: Mapping the Risks. Washington: World Resources Institute, 2003.

Mittermeier, R. A.; Robles Gil, P.; Hoffmann, M.; Pilgrim, J.; Brooks, T.; Mittermeier, C. G.; Lamoreux, J.; Da Fonseca, G. A. B. (Eds.). Hotspots revisited: Earth's biologically richest and most endangered terrestrial ecoregions. CEMEX/ Agrupación Sierra Madre, Mexico City. 392p, 2004.

Myers, N.; Mittermeier, R. A.; Mittermeier, C. G.; Da Fonseca, G. A.; Kent, J. Biodiversity hotspots for conservation priorities. Nature, 403, 853-858, 2000.

O’ Rourke, D.; Connolly, S. Just oil? The distribution of environmental and social impacts of oil production and consumption. Annual Review of Environment and Resources, 28, 587-617, 2003.

Osti, M.; Coad, L.; Fisher, J. B.; Bomhard, B.; Hutton, J. M. Oil and gas development in the World Heritage and wider protected area network in sub-Saharan Africa. Biodiversity and Conservation, 20(9), 1863-1877, 2011.

Ostrom, E. Understanding institutional diversity. Princeton,
NJ: Princeton University Press, 2005.

Piagentini, P. M.; Favareto, A. S. Instituições para regulação ambiental: o processo de licenciamento ambiental em quatro países produtores de hidreletricidade. Desenvolvimento $e$ Meio Ambiente, 30, 31-43, 2014.

Piquet, R. Os Efeitos Multiplicadores da Indústria Brasileira de Petróleo. Revista Paranaense de Desenvolvimento, 123, 81-97, 2012.

Piquet, R.; Serra, R. (Org.). Petróleo e região no Brasil: o desafio da abundância. Rio de janeiro: Garamond, 2007.

Reid, J. O. H. N.; Sousa JR, W. Investimentos em infraestrutura e políticas de conservação no Brasil. Megadiversidade, 1, 189-197, 2005.

Renn, O. The social arena concept of risk debates". In: Krimsky, S.; Golding, D. (Eds.). Social Theories of Ris. Praeger, Westport, CT, 1992.

Ribeiro, M. C.; Ribeiro, M. C.; Metzger, J. P.; Martensen, A. C.; Ponzoni, F. J; Hirota, M. M. The Brazilian Atlantic Forest: How much is left, and how is the remaining forest distributed? Implications for conservation. Biological Conservation, 142, 1141-1153, 2009.

Rougemont, L.; Pérez, M. S. Tecendo relações entre os conflitos socioambientais territoriais provocados por megaprojetos: Comperj e Suape e suas implicações para pescadores e pescadoras artesanais. Campo-Território, 8(16), 2013.

San Sebastián, M.; Hurtig, A. K. Oil exploitation in the Amazon basin of Ecuador: a public health emergency. Pan American Journal of Public Health, 15, 205-211, 2004.

São Paulo. Decreto $N^{o} 25.341$, de 04 de Junho de 1986. Aprova o Regulamento dos Parques Estaduais Paulistas.

São Paulo. Decreto $n^{\circ}$ 29.762, de 20 de Março de 1989. Acrescenta dispositivo ao Regulamento, aprovado pelo Decreto $n^{0}$ 25.341, de 4 de junho de 1986.

SMA - Secretaria do Meio Ambiente de São Paulo. Plano de Manejo do Parque Estadual da Serra do Mar. São Paulo: IF, 2006. 445p.

Simmel, G. Sociologia. Organização de Evaristo de Moraes Filho. São Paulo: Ática, 1983. 
Simões, E. O dilema das decisões sobre populações humanas em Parques. Campinas, (Tese) Doutorado em Ambiente e Sociedade-NEPAM-UNICAMP, 2010.

Souza, M. P. R. Análise da atuação de ONGs socioambientalistas no Litoral Norte de São Paulo. Trabalho de Conclusão de Curso. (Graduação em Ciências Biológicas) - Universidade Estadual Paulista, 2007.

Teixeira, L. MegaProjetos no Litoral Norte Paulista: O Papel dos Grandes Empreendimentos de Infraestrutura na Transformação Regional. Campinas, (Tese) Doutorado em Ambiente e Sociedade - IFCH-UNICAMP, 2013.

Unesco. Analytical summary of the state of conservation of World Heritage Properties. United Nations Educational Scientific and Cultural Organization, 2009. Disponível em: <whc.unesco.org/document/134872>. Acesso em: Abr. 2015.
Viglio, J. E. Usos sociais e políticos da ciência na definição de riscos e impactos ambientais no setor de petróleo e gás. Campinas, (Tese) Doutorado em Ciências Sociais. - IFCHUNICAMP, 2012

Zhouri, A.; Laschefski, K. Desenvolvimento e conflitos ambientais. Belo Horizonte: Ed. UFMG, 2010.

Zhouri, A.; Oliveira, R. Desenvolvimento, conflitos sociais e violência no Brasil rural: o caso das usinas hidrelétricas. Ambiente \& Sociedade, 10(2), 119-136, 2007. 